XXVI.

Aus der medicinischen Poliklinik zu Halle a. S.

\title{
Ueber die diabetische Acidose und ihre Beeinflussung durch Haferkuren.
}

\author{
Von \\ Dr. H. Jastrowitz, und Dr. H. Beuttenmüller, \\ derzeitirem Assistenten der Poliklinik ehemaligem Assistenten der Poliklinik.
}

Die im Jahre 1903 durch v. Noorden (1) eingeführte Haferkur gilt heute als allgemein anerkannter therapeutischer Factor bei der schweren Form des Diabetes. $\Lambda$ n den ursprünglichen Indicationen wird, wie aus den Abhandlungen von Lampé (2), Falta (3), Lüthje (4) erhellt, im Wesentlichen jetzt noch festgehalten. lis giebt Kranke, die allgemein günstig beeinflusst werden, solche, bei denen nur die Ketonurie oder die Glukosurie sich bessert und endlich einige, die sich in jeder Hinsicht refractär verhalten. Ferner gelingt es mitunter durch eine Haferkur, auch ohne dass Ketonurie besteht, die Toleranzgrenze zu heben, sowie sogar Kranke, bei denen andersartige Maassnahmen versagen, trot\% der hohen $\mathrm{K} \cdot \mathrm{H}-\mathrm{Zufuhr}$ auf diesem Wege zuckerfrei zu machen. Die Wirkung der Haferkur beruht einmal in dem besonderen, z. Th. noch ungeklärten Einfluss des Hafers, ferner auf der geringen Quantitüt des zugeführten Eiweisses, mit der eine gewisse Stickstoffunterernährung verknüpft ist, sowie endlich auf der Form des Pflanzen-bezw. Eierallumins, in der das Eiweiss gereicht wird. Gerade diese Eiweissarten verhalten sich im diabetischen Organismus in Bezug auf die Zuckerbildung im Allgemeinen am günstigsten, $d . h$. es findet aus ihnen die geringste Zuckerbildung statt [Lüthje (5), Mohr (6), Falta (7)]. Namentlich in Fällen von Eiweiss- wie von K·H-Ueberempfindlichkeit konnten auf diesem Wege Besserungen erzielt werden.

Immerhin sind die Dauererfolge doch recht wechselnde; zwar ist in einigen ganz schweren Fällen, so z. B. von Noorden (8) bei einem jugendlichen Diabetiker eine dauernde, weitgehende Besserung verzeichnet worden. Im Ganzen jedoch geben eine günstige Prognose, worauf schon Lampé hinweist, am ehesten die schweren Formen des mittleren Lebensalters. Ein definitives Urtheil abzugeben, ist häufig deshalb schwierig, weil eine längere exacte Beobachtung nicht überall durchführbar ist und man nach der lintlassung aus der Klinik bezüglich der Beobachtung diätetischer Vorschriften ausschliesshich von der Zuverlässigkeit des Pa- 
tienten abhängig ist. So können entweder anfängliche Besserungen als dauernde bezeichnet oder zunächst nicht günstig beeinflusste Kranke als ungeeignet für cinen solchen diätetischen Angriff angesehen werden. Hierzu kommt, dass schwere Diabetiker auf die einzelnen Diütformen individuell ganz verschicden reagiren: man ist hier zur Feststellung der geeigneten Therapie lediglich auf das Experiment am Krankenbette angewiesen. Erst auf diese Weise kann ferner entschieden werden, ob es sich in solchen Fällen wirklich um ganz schwere inkurable Formen handelt, oder nur um solche, die wir lediglich wegen des Bestehens der Ketonurie schulgemäss als schwere anzusehen gowohnt sind. In der Absicht, aus dem Verhalten einzelner Factoren der diabetischen Stoffwechselstörung vielleicht Hinweise auf die Prognose des Falles oder auf die Wirkungsweise der Diät, besonders des Hafers, zu erhalten, haben wir 3 sehwere Diabetiker einer länger dauernden klinischen Beobachtung unterzogen und Glukosurie, Ketonurie sowie die Vertheilung einiger N-haltiger Substanzen (Ammoniak, ITarnstoff, Rest-N) dabei verfolgt.

Bevor auf die Besprechungen im Einzelnen eingegangen wird, sei nur kurz angeführt, dass die Bestimmungen wach den üblichen Methoden gemacht wurden: $\mathrm{N}$ nach Kjeldahl, $\mathrm{NH}_{3}$ nach Krüger und Reich, Aceton nach Huppert-Messinger, Oxybuttersäure nach MagnusLevy, Oxalsäure nach Autenrieth und Barth, Harnstoff nach MörnerSjöqvist mit der lïr zuckerhaltige Harne nöthigen Modilication (Zusatz von Barythydrat).

Der /ucker wurde gravimetrisch (nach Soxhlet-Allihn) sowie polarimetrisch, an einzelnen Tagen auch durch Vergährung, bestimmt. Die Polarisationsresultate zeigten hänfig von den wahren Werthen starke Abweichungen, da der Urin an einzelnen Tagen infolge des geringen Zucker- und hohen Oxybuttersäuregehaltes nur eine sehr geringe Reehtsdrehung aufwies oder eine solche überhaupt vermissen liess. Im Gegensatz hierzu stiumen die durch Vergährung gewonnenen, in die Tabellen jedoch nicht aufgenommenen Resultate mit den durch Reduction ermittelten im Allgemeinen überein; auch liess die Vergährung besonders aufällige Erscheinungen nicht erkennen.

Für die Extraction der Oxybuttersäure bedienten wir uns des von Embden und Schmitz (9) empfohlenen Lindt'schen Apparates, von dessen Vorzügen gegenüber den früher gebräuchlichen, z. B. dem Schwarz'schen Apparat, wir uns überzeugen konnten. Es sei nur bemerkt, dass die Dauer der Extractionszeit wesentlich von der Umdrehungsgeschwindigkcit der Rührspindel abhängt und insofern der von uns angewandte Antrieb durch Wasserturbine nicht empfehlenswerth ist, weil diese, in Folge ihrer Abhängigkeit von den Schwankungen des Wasserdruckes, nicht immer mit gleicher Geschwindigkeit arbeitete, wodurch die Extractionszeit bis zu 12_-14 Stunden verlängert wurde. Ein zweiter Mangel ist die wenig ausgiebige Regulierbarkeit der Temperatur des Sandbades, ein Uebelstand, dem sich duroh Einschaltung eines Kurbel-Rheostaten leicht abhelfen liess.

An einigen Versuchstagen, die eine auffällige Differenz zwischen der Summe des $\mathrm{U}-\mathrm{N}$ und $\mathrm{NH}_{3}-\mathrm{N}$ einerseits, sowie dem Gesammt- $\mathrm{N}$ aufwiesen, ist dann bei 2 Patienten der Niederschlags-N bestimmt worden. Es wurde hierbei so vorgegangen, dass der Ammoniak, wie bei der Kr üger-Reich'schen Methode, durch Natriumearbonat im Vacuum ausgetrieben und der mit Salzsäure angesäuerte Harn mit 10 proc. Phosphorwolframsalzsäure gefällt wurde. Nach Absetzen des Niederschlages wurde derselbe mit der Fällungsflüssigkeit und dann mit Alkohol-Aether ausgewaschen und der Stick- 
staff des Niederschlages bestimmt. Es wurden für jeden Versuch $50 \mathrm{ccm}$ Urin verwandt und stets mehrere Controlen ausgeführt.

\section{Beobachtung I.}

In dem ersten Falle handelte es sich um einen poliklinischen $\mathrm{Pa}$ tienten $\mathbf{1}$ ).

Der 21 jährige Pat. Kurt H . . . datirt seine Beschwerden (Polydipsie, Mattigkeit, Abmagerung) 4 Jahre zurück. Der Diabetes wurde zuerst 1907 constatirt. Im Verlaufe der Krankheit stellten sich Kopfschmerzen ein, ferner traten Zahncaries, Haarausfall sowie öfter Furunkel, Juckreiz und Sprödigkeit der Haut auf. Seit einem halben Jahre vor der Aufnahme hatte sich das Allgemeinbefinden langsam aber stetig verschlechtert, auch die Sehkraft hatte abgenommen. Objectiv wurde an den inneren Organen des körperlich sehr mageren Kranken ausser einer leichten Affection der linken Lungenspitze ein wesentlicher Befund nicht erhoben. Dagegen wurden Ohnmachtsanwandlungen und in letzter 'Zeit eine zunehmende Schlafsucht beobachtet. Psychisch war er leicht reizbar, befolgte die diätetischen Maassnahmen nur widerwillig, so dass es bald zu einem Conflict kam, infolge dessen er die Klinik verliess. Pat. lebt zur Zeit noch, wie festgestellt werden konnte, ist jedoch diesseits nicht mehr beobachtet worden.

Tabelle I. (Curt H.)

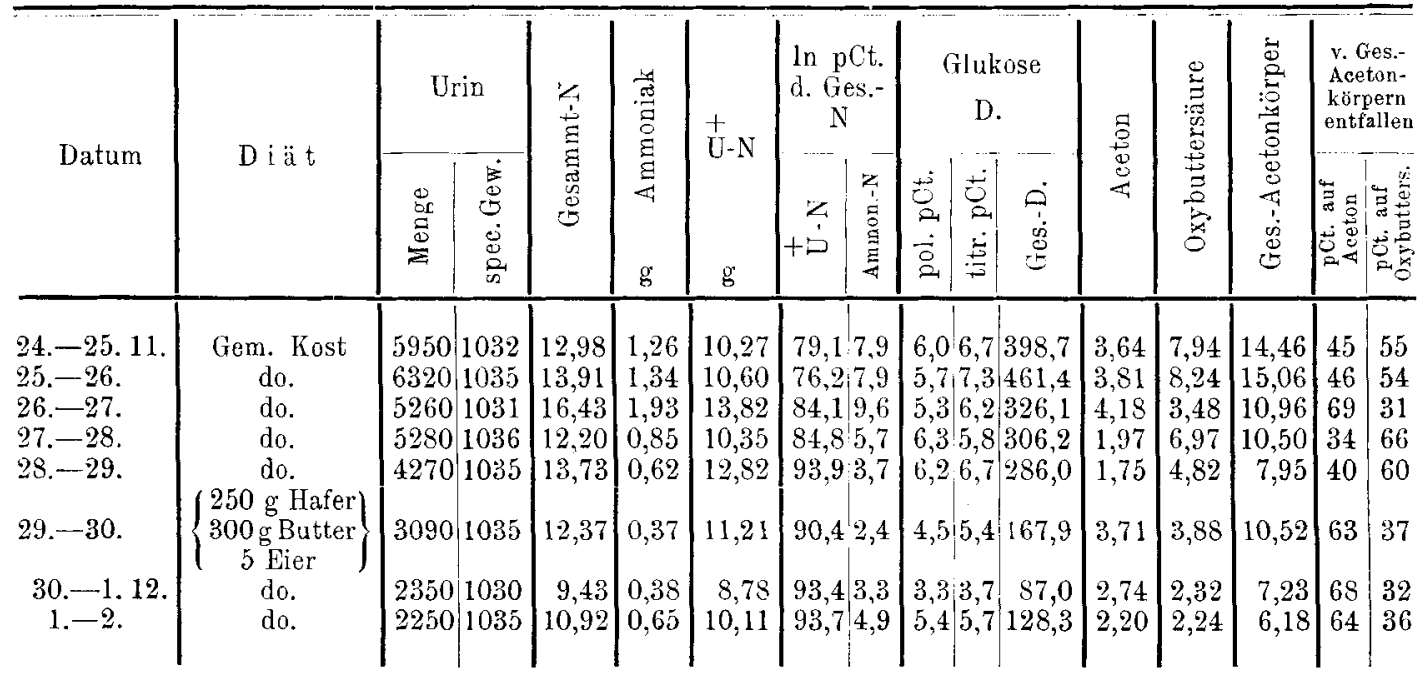

Tahelle II. (Curt H., Uebersicht.)

\begin{tabular}{c|c|c|c|c|c|c}
\hline Datum & Dï̈t & Ges.-N. & Ges.-D. & $\begin{array}{r}\text { Ges.- } \\
\text { Aceton- } \\
\text { körper }\end{array}$ & $\mathrm{NH}_{3}$ ing & $\begin{array}{c}\mathrm{NH}_{3} \text { in } \\
\text { pCt. des } \\
\text { Ges.-N. }\end{array}$ \\
\hline 24.-29. 12. & Gem. Kost & 13,85 & 355,7 & 11,79 & 1,22 & 7,9 \\
30.11. bis 2. 12. & Hafer & 10,03 & 127,7 & 7,98 & 0,47 & 3,5
\end{tabular}

1) Herm Geh.-Rath Schmidt, der uns die Beobachtung des Kranken in seiner Klinik gestattete, sind wir hierfür zu Dank verpflichtet. 
Während der ersten Beobachtungstage wurde dem Patienten die gewöhnliche Kost verabfolgt: die Zuckerausseheidung (ef. Tab. I und II), die am ersten Tage $398,7 \mathrm{~g}$, am zweiten $461,4 \mathrm{~g}$ betrug, sank ohne jegliche Diätbeschränkung am fünften Tage auf $286 \mathrm{~g}$ bei annähernd gleichbleibender Stickstoffausscheidung. Derselbe Einfluss zeigt sich auf die Ketonurie. Die Gesammtausscheidung der Acetonkörper betrug $8 \mathrm{~g} \mathrm{am}$ fünften Tage gegenüber $14-15 \mathrm{~g}$ bei der Aufnahme.

Der Haferkur, welcher er unterworfen werden sollte, entzog der Kranke sich, wie schon angeführt, sehr bald, so dass unsere weitere Beobachtung sich nur auf 3 Tage erstreckt. Am letzten Beobachtungstage ging trotz Zufuhr von $180 \mathrm{~g}$ Kohlehydrat der Zucker bis auf $128,3 \mathrm{~g}$ herab. Ein entsprechender Abfall der Acidose liess sich bei der Kürze der Beobachtung nicht constatiren; sie bewegte sich in den letzten 5 Tagen ca. zwischen 6 und $11 \mathrm{~g}$ Oxybuttersäure. Auffallend erscheint endlich, dass während der Hafertage die N-Ausscheidung nicht entsprechend der Verminderung der N-Zufuhr herabgeht (13,85 gegen 10,05 g).

Dieser Fall zeigt, wie sehr Schonung allein den Zustand eines Diabetikers mitunter günstig beeinflussen kann; daher wäre es vielleicht

Tabelle III

\begin{tabular}{|c|c|c|c|c|c|c|c|c|c|}
\hline \multirow[b]{2}{*}{ Datum } & \multirow{2}{*}{\multicolumn{2}{|c|}{$\mathrm{Nahrung}$}} & \multicolumn{2}{|c|}{ Urin } & \multirow[b]{2}{*}{ Ges.- $\mathrm{N}$} & \multirow[b]{2}{*}{$\mathrm{NH}_{3}$} & \multirow{2}{*}{$\mathrm{U} \cdot \mathrm{N}$} & \multicolumn{2}{|c|}{$\begin{array}{l}\text { In pCt. des Ge- } \\
\text { sammt- }\end{array}$} \\
\hline & & & 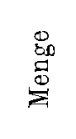 & $\begin{array}{l}\dot{E} \\
\stackrel{0}{0} \\
\dot{0} \\
\dot{0} \\
\tilde{D}_{\infty}\end{array}$ & & & & 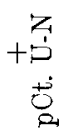 & 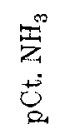 \\
\hline $25 .-26.11$. & & & 2450 & 1,036 & 13,15 & 1,82 & 10,87 & 82,4 & 11,3 \\
\hline $26 .-27$. & Strenge Diät + & & 2910 & 1,039 & 12,14 & 1,59 & 10,25 & 84,7 & 10,8 \\
\hline $27 .-28$ & $300 \mathrm{~g}$ Weissbrod & .7 & 3000 & 1,035 & 15,38 & 1,81 & 12,82 & 83,2 & 9,6 \\
\hline $28 .-29$. & $750 \mathrm{~g}$ Hafer & & 1970 & 1,033 & 7,32 & 0,96 & 6,27 & 85,9 & 10,8 \\
\hline $29 .-30$. & $600 \mathrm{~g}$ Butter & & 1690 & 1,030 & 5,99 & 0,80 & 5,02 & 83,7 & 11,2 \\
\hline $30.11 .-1.12$ & 0 & & 1570 & 1,022 & 8,02 & 1,25 & 6,41 & 80,1 & 12,9 \\
\hline 1. -2.12 . & Gemüse + & & 1250 & 1,033 & $7,7 \mathrm{~S}$ & 0,51 & 6,27 & 80,4 & 5,4 \\
\hline $2 .-3$. & $900 \mathrm{~g}$ Butter & . & 950 & 1,029 & 7,94 & 0,42 & 6,69 & 84,7 & 4,3 \\
\hline $3,-4$. & +24 Fier & & 1580 & 1,028 & 7,82 & 0,41 & 6,63 & 85,0 & 4.3 \\
\hline $4 .-5$ & f Strenge Diat + & & 2200 & 1,033 & 14,91 & 0,57 & 13,79 & 92,1 & 3,2 \\
\hline $5 .-6$. & $\{40 \mathrm{~g}$ Weissbrod & $.\}$. & 2100 & 1,033 & 16,88 & 0,66 & 15,78 & 89,8 & 3,2 \\
\hline 6. -7 . & $1000 \mathrm{~g}$ Hafer & & 2520 & 1,021 & 8,33 & 0,69 & 6,56 & 79,0 & 6,9 \\
\hline 7. -8 & $+800^{\circ}$ Butter & & 2350 & 1,026 & 8,45 & 0,68 & 6,73 & 79,2 & 6,6 \\
\hline 8. -9 & & & 2410 & 1,026 & 8,92 & 1,15 & 6,93 & 77,8 & 10,6 \\
\hline $9 .-10$. & & & 2750 & 1,021 & 8,25 & 0,53 & 6,78 & 73,7 & 4,7 \\
\hline $10 .-11$. & Gemüse $+1200 \mathrm{~g}$. & & 1960 & 1,023 & 8,26 & 0,37 & 7,02 & 84,6 & 3,6 \\
\hline $11 .-12$. & | Butter +32 Eier. & & 1530 & 1,021 & 6,18 & 0,30 & 5.23 & 84,3 & 4,0 \\
\hline $12 .-13$ & $+160 \mathrm{~g}$. & & 1610 & 1,015 & 5,30 & 0,34 & - & - & - \\
\hline $13 .-14$ & Weissbrod & & 1880 & 1,016 & 5,32 & 0,50 & - & - & 一 \\
\hline $14,-15$ & Gemüse & & 1500 & 1,020 & 6,74 & 0,43 & 6,13 & 91,5 & 5,2 \\
\hline $15-16$ & $\int+1200 \mathrm{~g}$ Butter & & 1940 & 1,016 & 8,04 & 0,38 & 6,72 & 84,0 & 3,9 \\
\hline $16 .-17$. & +20 Eier. . & & 2200 & 1,017 & 7,04 & 0,57 & 6,41 & 91,6 & 6,7 \\
\hline $17 .-18$ & . . . . & & 2360 & 1,013 & 7,83 & 0,42 & 7,38 & 94,6 & 4,4 \\
\hline 18 & Gemüse +90 & & 1960 & 1,020 & 9,68 & 0,25 & 8,96 & 92,3 & 2,2 \\
\hline 19 & Butter +15 Eier & & 2450 & 1,021 & 13,54 & 0,25 & 12,90 & 95,6 & 1,6 \\
\hline $20 .-21$. & $+450 \mathrm{~g}$ Fleisch & & 1210 & 1,017 & 13,02 & 0,16 & 11,91 & 91,6 & 1,2 \\
\hline
\end{tabular}


zweckmässig, so weit dies ohne periculum vitae ausführbar ist, Patienten, wie hier geschehen, zunächst nnter günstige äussere Bedingungen ohne jegliche spezielle Kostform zu setzen und erst auf Grund einer weiteren Beeinflussung durch diätetische Maassnahmen den therapeutischen Werth letzterer einzuschätzen.

\section{Beobachtung $\mathrm{II}^{\mathbf{1}}$ ).}

In dem zweiten Falle handelte es sich um einen 46jährigen Lehrer Ernst F. Diabetes vor 2 Jahren festrestellt. Nach einer diätetischen Kur angeblich vorübergehend zuckerfrei. Erst im Laufe des letzten Jahres rapide Verschlechterung des Befindens: Patient bemerkte eine starke Herabsetzung seiner körperlichen Leistungsfähigkeit und rasche geistige Ermüdbarkeit bei Ausübung seines Berufes. Er hatte im Laufe des letzten Jahres ca. $10 \mathrm{~kg}$ abgenommen; es stellte sich heftiges Hautjucken ein; neuralgiforme, namentlich Nachts exacerbirende Schmerzen in den Beinen, hauptsächlich "an den Aussenseiten beider Oberschenkel, raubten ihm häufig den Schlaf. Durst nicht gesteigert, dagegen leidet Pat, an abnormem Hungergefühl.

1) Fall II und III betrafen Patienten, die in der Privatklinik im Einzelzimmer bei Bettruhe beobachtet wurden.

(Ernst F.).

\begin{tabular}{|c|c|c|c|c|c|c|c|c|c|}
\hline $\begin{array}{l}\dot{8} \\
8 \\
\dot{\Xi} \\
0\end{array}$ & 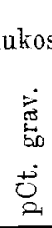 & 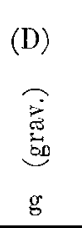 & $\frac{\mathrm{D}}{\mathrm{n}}$ & $\begin{array}{l}\stackrel{a}{\circ} \\
\stackrel{8}{8} \\
\stackrel{\rho}{4}\end{array}$ & 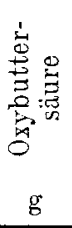 & 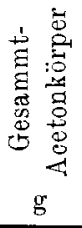 & 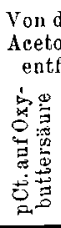 & 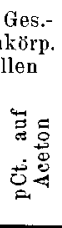 & Bemerkungen. \\
\hline 5,6 & 6,3 & 154,4 & $17,7: 1$ & 2,02 & 9,26 & 12,88 & 29 & 71 & tgl. abends 0,5 Aspirin. \\
\hline 4,4 & 6,7 & 195,0 & $16,1: 1$ & 2,25 & 9,02 & 13,06 & 31 & 69 & Oxalsäure $5,3 \mathrm{mg}$. \\
\hline 5,0 & 6,0 & 180,0 & $11,7: 1$ & 2,10 & 5,40 & 9,00 & 40 & 60 & do. 5,7 \\
\hline 4,8 & 5.6 & 110,1 & $16,5: 1$ & 1,35 & 4,89 & 7,32 & 33 & 67 & do. \\
\hline 5,1 & 5,3 & 89,1 & $14,9: 1$ & 1,23 & 2,24 & 4,50 & 50 & 50 & 21,4 \\
\hline 3,9 & 4,7 & 73,8 & $8,2: 1$ & 1,31 & 1,41 & 3,75 & 62 & 38 & $\begin{array}{l}\text { Gew. } 64,6 \text {. Oxalsäure } 7,5 \mathrm{mg} \text {. } \\
\text { Niederschlags - N } 4,9 \text { pCt. }\end{array}$ \\
\hline 2,3 & 3,7 & 48,3 & $6,2: 1$ & 1,33 & 1,61 & 3,99 & 60 & 40 & $\begin{array}{l}\text { Gew. } 64,1 \text { Oxalsäure } 13,0 \mathrm{mg} \text {. } \\
\text { Niederschlags }-\mathrm{N} 8,8 \mathrm{pCt} .\end{array}$ \\
\hline 0,8 & 1,3 & 12,4 & $1,6: 1$ & 0,89 & 0,00 & 1,53 & 100 & 0 & Oxalsüure $9,4 \mathrm{mg}$. \\
\hline 0,9 & 1,7 & 26,9 & $3,4: 1$ & 1,37 & 0,00 & 2,45 & 100 & 0 & - \\
\hline 1,3 & 2,0 & 44,0 & $2,9: 1$ & 3,07 & 1,98 & 7,48 & 73 & 27 & Gew. 65,0 . \\
\hline 2,5 & 3,3 & 69,3 & $4,1: 1$ & 1,74 & 9,00 & 12,11 & 26 & 74 & - \\
\hline 2,5 & 3,0 & 75,6 & $9,1: 1$ & 2,08 & 6,36 & 10,08 & 37 & 63 & Niederschlags - N 9,3 pCt. \\
\hline 3,4 & 3,6 & 84,6 & $9,9: 1$ & 2,43 & 2,82 & 7,17 & 60 & 40 & Gew. 63,9. Niederschlags - N 10,7 pCt. \\
\hline 3,3 & 3,4 & 81,7 & $9,1: 1$ & 0,77 & 1,08 & 2,46 & 56 & 44 & Niederschlags - $\mathrm{N} 9,7 \mathrm{pCt}$. \\
\hline 2,6 & 2,9 & 79,8 & $8,6: 1$ & 0,91 & 1,44 & 3,07 & 53 & 47 & - \\
\hline 1,1 & 1,2 & $23, \check{5}$ & $2,8: 1$ & 0,47 & 0,00 & 0,84 & 100 & 0 & Niederschlags-N 8,3 pCt. \\
\hline 0,7 & 1,0 & 15,3 & $2,4: 1$ & 0,22 & 0,00 & 0,39 & 100 & 0 & Gew. 64,2 . Niederschlags $-\mathrm{N} 7,1 \mathrm{pCt}$. \\
\hline 0,4 & 0,8 & 12,9 & $2,4: 1$ & 0,23 & 0,00 & 0,39 & 100 & 0 & - \\
\hline 1,0 & 1,5 & 28,2 & $5,3: 1$ & 0,36 & 0,00 & 0,64 & 100 & 0 & - \\
\hline 1,5 & 1,5 & 22,5 & $3,3: 1$ & 0,44 & 0,00 & 0,79 & 100 & 0 & Niederschlags-N 2,2 pCt. \\
\hline 0,5 & 0,8 & 15,5 & $1,9: 1$ & 1,37 & 2,20 & 4,65 & 52 & 48 & Gew. $64,2 . \quad$ Niederschlags $-\mathrm{N} 9,9 \mathrm{pCt}$. \\
\hline 0,2 & 0,4 & 8,8 & $1,3: 1$ & 0,80 & 0,75 & 2,18 & 66 & 34 & 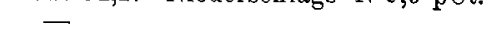 \\
\hline 0,3 & 0,5 & 11,8 & $1,6: 1$ & 0,73 & 1,72 & 3,03 & 43 & 57 & - \\
\hline 0,4 & 0,9 & 17,6 & $1,8: 1$ & 1,06 & 2,08 & 3,98 & 48 & 52 & Gew. 63,7 . \\
\hline 0,7 & 1,3 & 31,9 & $2,4: 1$ & 0,95 & 0,00 & 1,70 & 100 & 0 & - \\
\hline 0,9 & 1,4 & 16,9 & $1,3: 1$ & 0,57 & 0,43 & 1,54 & 70 & 50 & Gew. 64,3. \\
\hline
\end{tabular}


Objectiver Befund: Mässig genährter Mann von blassem Aussehen; Haut und innere Organe ohne wesentlichen Befund. Die Pupillen reagiren prompt, Hautund Sehnenreflexe sind vorhanden, nur die Patellarrellexe sind herabgesetzt, die Motilität ist ungestört; an der Aussenseite der beiden Oberschenkel ist die Berührungsund Schmerzempfindung beiderseits herabgesetzt, entsprechend dem Ausbreitungsgebiet des N. cutaneus femoris lateralis. Im Uebrigen ist die Sensibilität intakt. Urin ohne Eiweiss, reichlich Acetessigsïure und Zucker.

Pat., dessen Befinden durch klinische Behandlung sich wesentlich besserte, bekam ca. 1 Monat nach der Entlassung einen Karbunkel und im Anschluss hieran Cona, dem er rasch erlag.

Dieser Pat. wurde ohne jegliche andere Medication als $0,5 \mathrm{~g}$ Aspirin, die er wegen der besprochenen neuritischen Schmerzen erhielt, 26 Tage von uns beobachtet. Es sind bei ihm 2 Haferperioden, 3 Gemüseperioden, daron eine nit Brod, die andere mit Wleischzulage, sowie 2 Perioden mit strenger Diät, allerdings unter Brodzulage, beobachtet worden. Der Uebersichtlichlieit halber und weil die Nahrungsaufnahme nicht an jedem 'lage einer Diätperiode gleich gross war, ist hier die Summe aller während einer einzelnen Periode genossenen Speisen, obne Rücksicht anf die Grösse der Nahrungsaufnahme am einzelnen l'age zusammengefasst worden.

Zunächst zeigt die (ef. Tab. III und IV) Vertheilung der stickstoffhaltigen Bestandtheile des Urins doch ciniges Beachtenswerthe. Die Ammoniakausscheidung geht zwar im Allgemeinen der Curve der Gesammtacetonkörper parallel. In einzelnen Tagen sieht man jedoch, dass die Ammoniakausscheidung relativ gegenüber der des Cresammtstickstoffs erhöhte Werthe zeirgte: so während der ersten Haferperiode durchschnittlich 11,5 pCt., trotzdem die Acetonkörper gegenüber der ersten Periode (strenge Diät mit Brodzulage) von 11,6 auf $5,2 \mathrm{~g}$ heruntergegangen waren: allerdings möchten wir betonen, dass entsprechend der geringeren $\mathrm{N}-\mathrm{Zu}-$ fuhr nur $3,1 \mathrm{~g} \mathrm{~N}$ ausgeschieden wurden. Etwas auffällig crscheint ferner die Vermehrung des Niederschlags- $\mathrm{N}$ mit 9,3 pCt. und 10,7 pCt. zu Ungunsten des Harnstoff- $\mathrm{N}$ und Ammoniak- $\mathrm{N}$ während der zweiten Haferperiode.

Tabelle IV (Ernst F., Uebersicht).

\begin{tabular}{|c|c|c|c|c|c|c|}
\hline Datum & Di i & Ges. -N & Ges.-D & $\begin{array}{l}\text { Ge- } \\
\text { sammt- } \\
\text { Aceton- } \\
\text { körper }\end{array}$ & $\begin{array}{l}\mathrm{NH}_{3} \\
\text { in } \mathrm{g}\end{array}$ & $\begin{array}{l}\mathrm{NH}_{3} \text { in } \\
0 \% \text { des } \\
\text { Ges.-N }\end{array}$ \\
\hline $25 .-28.11$. & Strenge Diät $+57 \mathrm{~g}$ K. H. & 13,49 & 143,1 & 11,64 & 1,74 & 10,6 \\
\hline 28. $11 .-1.1$ & Hafer, $175 \mathrm{~g}$ K. H. & 7,11 & 91,0 & 5,1 & 1,00 & 11,5 \\
\hline 1. & liemii & & & & 0,43 & 4,5 \\
\hline-6.12 & Strenge Diät $+23 \mathrm{~g} \mathrm{~K}$. H. & 15,90 & 56 & 9,80 & 0,62 & 3,2 \\
\hline 1019 & 40 & 8,49 & 80 & 4,7 & 0,77 & 7,4 \\
\hline $10 .-14.12$ & $\begin{array}{c}\text { Gemüse }+40 \mathrm{~g} \mathrm{~K} . \mathrm{H} . \\
\text { (Weissbrod) }\end{array}$ & 6,27 & 20,0 & 0,57 & & ( \\
\hline $\begin{array}{l}14 .-18.12 \\
18 .-20.12\end{array}$ & $\begin{array}{c}\text { (iemüse, } 17 \mathrm{~g} \mathrm{~K} . \mathrm{H} . \\
\text { Gemüse + Fleisch, } 17 \mathrm{~g} \mathrm{~K} . \mathrm{H} .\end{array}$ & $\begin{array}{r}7,41 \\
12,08\end{array}$ & $\begin{array}{l}14,7 \\
22,1\end{array}$ & $\begin{array}{l}2,66 \\
2,41\end{array}$ & $\begin{array}{l}0,45 \\
0,22\end{array}$ & $\begin{array}{l}5,0 \\
1,5\end{array}$ \\
\hline
\end{tabular}

Die Zuckerausscheidung, die bei strenger Diät mit Brodzulage 143,1 g betragen hatte, sank trotz erhöhter Kohlehydratzufuhr während der erston Haferperiode successive jeden Tag bis auf 73,8 g und unter Schwankungen während einer darauffolgenden Gemüseperiode auf $26,9 \mathrm{~g}$. Die dem Kranken versuchsweise dargereichte strenge Diät mit Brodzulage beant- 
wortete er mit einer Steigerung bis auf $69,3 \mathrm{~g}$ am 2. Tage dieses Regimes. Eine zweite 4 tägige Haferkur vermochte die Zuckerausscheidung nicht herabzudrücken, jedoch war die ausgeschiedene Menge auch hier immer geringer, als den in den Haferperioden zugeführten Kohlehydraten entsprach.

Einen besseren Erfolg zeigte eine darauf folgende Gemüseperiode mit Brodzulage, wo die Zuckerausscheidung auf $20 \mathrm{~g}$, und cine reine Gemüseperiode, wo sie auf $14,7 \mathrm{~g}$ abfiel. Einige zum Schluss eingeschaltete Tage mit Gemüse und Fleischzulage liessen den durchschnittlichen Zuckerwerth wiederum ansteigen. Aus dem Ausgeführten geht hervor, dass hier an eine Zuckerabspaltung aus Eiweiss zu denken ist. Hierauf deutet die letzte Periode hin, sowie vor allem die Tage strenger Diät (4.-6. Dec.), wo weit mehr Zucker ausgeschieden wurde, als den zugeführten Kohlehydraten entsprach. Eine wesentliche Toleranz für Kohlchydrate bestand hier nur während der Haferperiode, wo ca. nur die Hälfte der eingeführten Kohlehydrate als Glukose ausgeschieden wurde. Immerhin war dasselbe auch bei einer Zulage in Form von $40 \mathrm{~g}$ Weissbrod während einer Gemüseperiode der Fall.

Die Gesammtacetonkörperausscheidung, die in diesem Falle keine hohe war - der höchste Werth betrug 13,06 g am 2. Tage — sank sehr rasch bis auf Werthe herab, die wir, wie aus der erwähnten Mittheilung von Embden und Sehmitz hervorgeht, nicht mit Sicherheit als Oxybuttersäure ansprechen können, da die Bestimmung hier allein auf der Linksdrehung des Aetherextractes beruht. Eine Ausnahme macht nur die versuchsweise eingeschaltete Periode stronger Diät, wo $9,8 \mathrm{~g}$ Oxybuttersäure ausgeschieden wurden.

Das Verhältniss der ausgeschiedenen Oxybuttersäure zum Aceton gestaltet sich hier so, dass, je grösser die Oxybuttersäuremenge ist, um so geringer der Antheil des Acetons an der Summe der Gesammtacetonkörper. Irgend ein constantes Verbältniss zwischen Aceton und Oxybuttersäure liess sich nicht feststellen. Nur einige Zahlen erscheinen auffällig, so z. B. Acetonausscheidungen von 3,07 oder $2,43 \mathrm{~g}$ (4./5. und 8./6. 12) bei einer Ausscheidung von nur Spuren von Oxybuttersäure, sowie umgekehrt von nur $1,7 \mathrm{~g} \Lambda$ ceton und $9 \mathrm{~g}$ Oxybuttersäure an einem dazwischen gelegenen Tage (5./6. 12.). Dies lässt an eine Reciprocität bei der Entstehung der Acetonkörper denken, wie dies Neubauer (10) an einigen Fälen nachweisen konnte.

\section{Beobachtung III.}

Der 3. Fall ist der schwerste und am längsten beobachtete. $\mathrm{Zu-}$ nächst wollen wir die klinischen Daten vorausschicken.

Die 20jährige Erna F . . . war vor einem Jahre wegen langandauernder Zahneiterung (Alveolarpyorrhoe) in Behandlung; da im Laufe der letzten 2 Jahre die Zähne alle sebr schlecht geworden waren, wurde gelegentlich der zahnäzztlichen Be. handlung der Urin untersucbt und Zucker darin gefunden. Kurz vor der Entdeckung des Zuckers war Pat. durch Ausgleiten auf der Treppe auf den Kopf gefallen; dieses Trauma bringt sie in zeitlichen Connex mit der sehr starken Gewichtsabnahme der 
Tabelle V

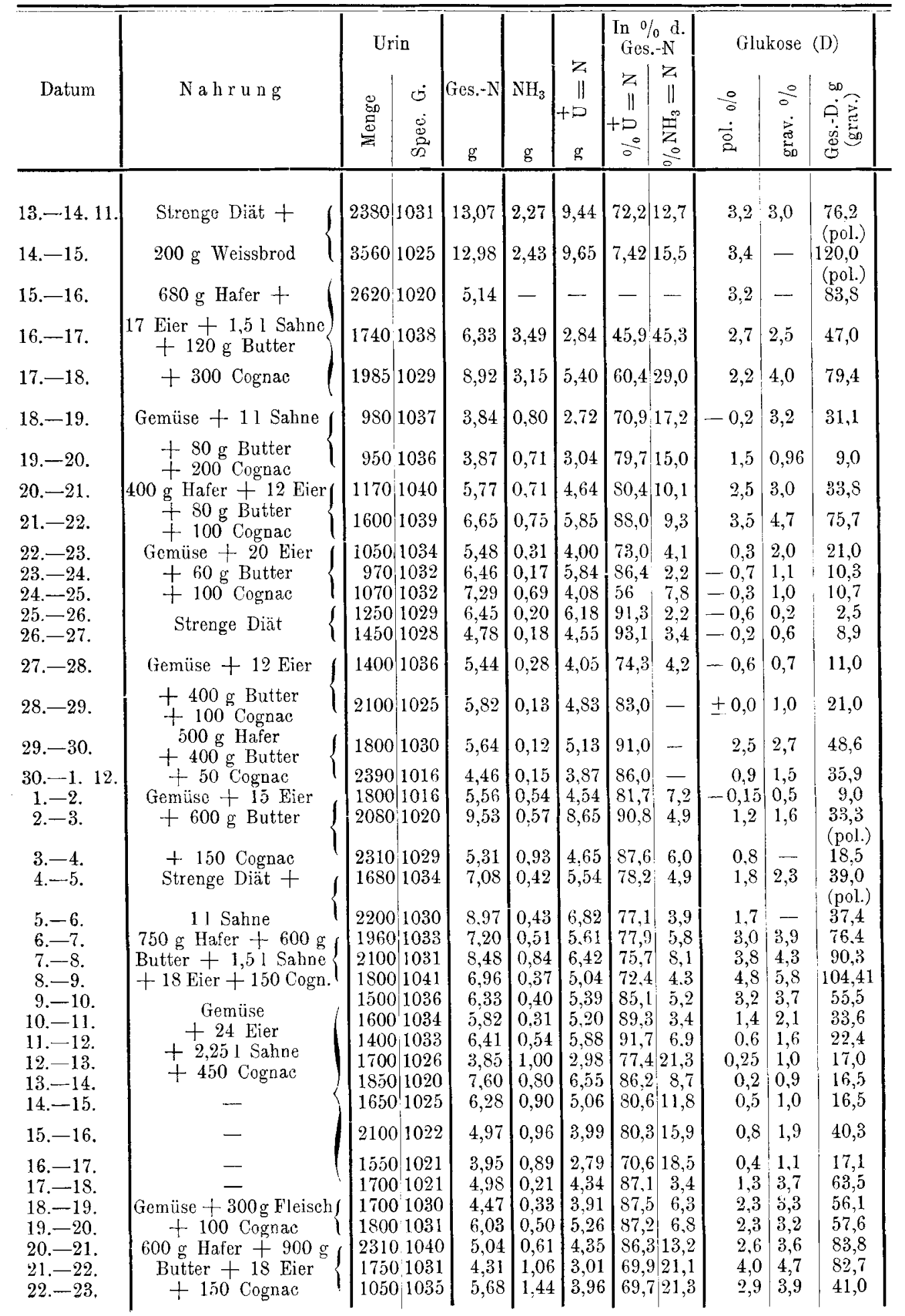


(Erna F.).

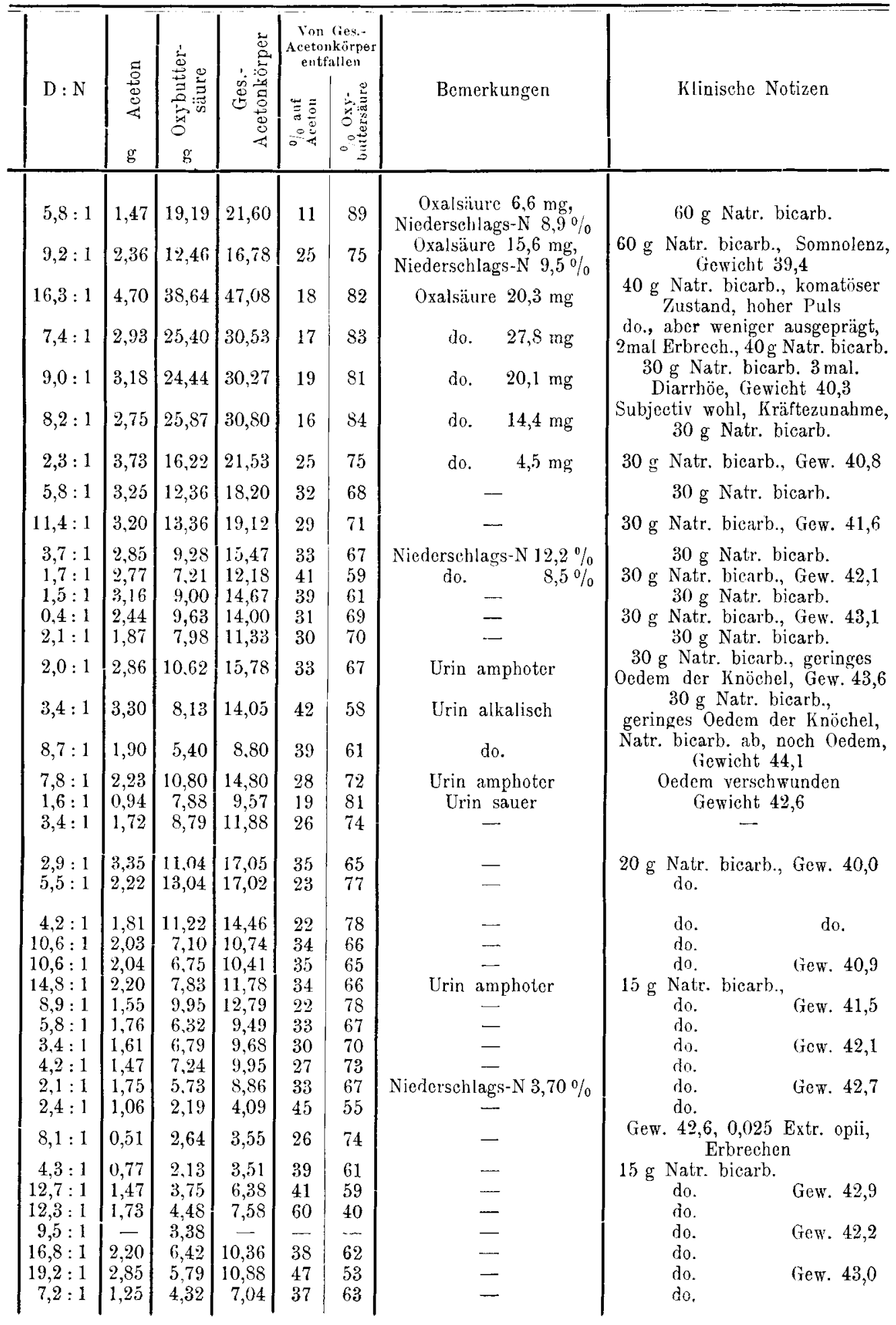


Tabelle VI (Erna F., Uebersicht).

\begin{tabular}{|c|c|c|c|c|c|c|}
\hline Datum & D i ät & Ges.-N & Ges. - D & $\begin{array}{l}\text { Gie- } \\
\text { sammi- } \\
\text { Aceton- } \\
\text { körper }\end{array}$ & $\begin{array}{l}\mathrm{NH}_{3} \\
\text { in } \mathrm{g}\end{array}$ & $\begin{array}{l}\mathrm{NH}_{3} \text { in } \\
0 \% \text { des } \\
\text { Ges.- } \mathrm{N}\end{array}$ \\
\hline $13 .-15.11$ & Strenge Diät, $57 \mathrm{~g}$ K. $1 \mathrm{I}$. & $1 ;, 03$ & 98,1 & 19,19 & 2,35 & 14,7 \\
\hline 15 & ( & & & & 3,32 & 39,0 \\
\hline $18 .-20.1$ & Gemüse, $20 \mathrm{~g} \mathrm{~K}$ K. H. & $\ddot{8}, 86$ & 20 & 26 & 0,76 & 15,8 \\
\hline $20 .-22.1$ & Hafer, $168 \% \mathrm{~K} . \mathrm{H}$. & 6,21 & 54 & 18, & 0,73 & 9,7 \\
\hline $22 .-25.11$. & I. H. & 6 , & 1. & 14. & 0,39 & 5,0 \\
\hline $25 .-27.1$ & Strenge Diat, $0 \mathrm{~g}$ K. II. & 5,62 & 5,7 & 12,67 & 0,19 & 2,7 \\
\hline 27 & Gemüse, $17 \mathrm{~g} \mathrm{~K} . \mathrm{H}$. & 5 , & 16,0 & 14,92 & - & for \\
\hline $29.11 .-1.12$ & Hafer, $176 \mathrm{~g} \mathrm{~K}$. H. & 5,05 & 42,3 & 11,8 & - & - \\
\hline $1 .-4.12$. & $17^{\circ} \mathrm{KK}$ & & & 12 & 0,68 & 7,9 \\
\hline $4 .-6.12$. & Strenge Diät, $4 \mathrm{~g} \mathrm{~K}$. ll. & 8,03 & 38 & 15, & 0,43 & 4,4 \\
\hline $6 .-9.1$ & er, $177 \mathrm{~g} \mathrm{~K}$ K. H. & & & 10 & 0,37 & 4,0 \\
\hline 9. -18.12 . & Gemüsc, $18 \mathrm{~g} \mathrm{~K}$. H. & 5,5 & 31 & 7,70 & 0,67 & 9,9 \\
\hline 18.-20. 12 . & Gemüse + Floisch, $17 \mathrm{~g} \mathrm{K.} \mathrm{H.}$ & 5,2 & 56 & - & 0,42 & 6,6 \\
\hline $20 .-23.12$ & Hafer, $140 \mathrm{~g}$ K. II. & 5,0 & 69,2 & 9,43 & 1,04 & 16,4 \\
\hline
\end{tabular}

letzten Zeit. In den letzten Wochen hatte Pat. auf ärztliche Verordnung hin eine strenge Diät ( $\Lambda$ usschluss von Kohlehydraten) erhalten.

Objectiver Befund: Blass, sebr mager, Haut trocken, welk, keine Oedeme, Zähne stark cariös, Alveolarpyorrhoe, dentlicher Acetongeruch der Atemluft. Ueber der rechten Spitze Schallverkïrzung, vorn wie hinten. Ebenso links vorn oben bis zur zweiten Rippe. Boiderseits feuchtes Rasseln. Herz nicht verbreitert, 'Töne rein, Puls 96, klein und wenig gespannt. Abdomen ohne Besonderheiten. Stuhl normal, keinerlei Motilitäts- noch Sensibilitätsstörungen, Patellarrellexe beiderseits nur schwach auslösbar.

Pat. wurde von Mitte November bis Lndo December von uns beobachtet und war dann noch bis Anfang Januar in der Klinik. Sie blieb nach ihrer Entlassung in Beobachtung, ohne dass die Zuckermenge zunächst wesentlich anstieg oder bedrohliche Symptome eintraten.

Naoh ca. 14 T'agen, im Anschlusse an eine Familienfestlichkeit, erkrankte sie plötzlich unter Erbrechen, allgemeiner Mattigkeit und Schlafsucht. Der Zustand verschlimmerte sich rasch und die Kranke erlag in 2 T'agen dem Coma.

Unsere Beobachtung setat in einem Zeitpunkt ein, wo die Kranke wochenlang hindurch sogenannte strenge Diät erhalten hatte. Die ersten Tage des Aufenthaltes wurden dazu benutzt, ihr zunächst die bisherige strenge Diät unter Brodzulage zu verabfolgen. Der Erfolg war der, dass Pat. zunehmend somnolent wurde und am 3. Tage, wo die eigentliche Behandlung durch Haferkur einsetzte, komatöse Erscheinungen bekam. Der schwere Zustand der Kranken liess es nicht zu, sie, wie den vorigen Fall, ohne Natr. bicarbonicum zu behandeln. Fs wurde ihr dasselbe zunächst in reichlicher Menge $(60 \mathrm{~g})$ verabfolgt; später, nach Schwinden der komatösen Erscheinungen, in successive fallenden Dosen bis zur Alkalescen\% des Urins. Von da ab wurde, ohne dass, wie wir weiter unten sehen werden, die Aeidose völlig sehwand, $15-20 \mathrm{~g}$ Natr. bicarbonicum wïhrend der ganzen Dauer der Beobachtung verabfolgt. In Folge dieses durch das Einsetzen des Coma nothwendig gewordenen medicamentösen Eingriffs wurde natürlich die Beurtheilung der einzelnen diätetischen Maassuahmen sowie die Verwerthung einzelner Factoren hierfür $\left(\mathrm{NH}_{3}\right)$ 
sehr erschwert. Sonst erhielt Pat. nur einmal Opium, das sie sehr bald erbrach, so dass hierdurch der Stoffwechsel wohl nicht becinflusst sein dürfte. Bei der $\Lambda$ ufzeichnung der Diät ist wie im Fall Il verfahren.

Was zunächst die Ausscheidung der stickstoffhaltigen Substanzen im Urin betrifft, so war das Verhältniss des Ammoniak- und des Harnstoffstickstoffes zu einander im Wesentlichen abhängig von der Grösse der Acidose und von dem allmählich eintretenden Ersatz des an Oxybuttersäure und $\Lambda$ cetessigsäure gebundenen $\Lambda$ mmoniaks durch das der Kranken verabfolgte Natron. Die Ammoniakausscheidung war natürlich während des Comas am höchsten; der Ammoniak-N entsprach mit $45 \mathrm{pCt}$ dem Harnstoff-N. Es ist dies ein Werth, der das von von Noorden (11) beschriebene relative Maximum der Ammoniakbildung beim Diabetes darstellt. Sie sank dann allmählich bis auf minimale Werthe $(2,7 \mathrm{pCt}$. pro Tag) mit der zunehmenden Alkalescenz des Urins herab. Nach Weglassen des Natrons und bei geringen Gaben desselben stiegen die Ammoniakwerthe um ein Weniges und erreichten nur an einzelnen lagen den Werth von $1 \mathrm{~g}$. Procentual war trotz der geringen Stickstoffausfuhr der Procentsatz des Ammoniak- $\mathrm{N}$ ein etwas höherer. An einzelnen Tagen erreicht er indessen wohl eine pathologische llöhe mit 18,5 pCt. (16. 11. 17. 12.) und an den beiden Endtagen der Beobachtung (21.-23. 12.) mit 21 pCt. des Gesammt-N. Nitunter zeigt auch der Niedersehlags-N abnorme Werthe, die am Schluss eingehender discutirt werden. Während der l'eriode der strengen Diät bei der Kinlieferung (13. 12.) betrug derselbe 8,9 und 9,5 pCt. des Gesammt-N. Obwohl sich ein gleiches Verhalten bei den weiteren Perioden strenger Diät nicht deutlich zeigt, so dürfte es doch lediglich auf die hohe Fleischration zurückzuführen sein. Auffälliger erseheint diese Steigerung des dysoxydablen Stickstoffs [Spiro (12)] an zwei Gemüsetagen (22.--24. 11.) mit 12,2 bzw. 8,5 pCt. Gesammt-N.

Trotz der Schwere des vorliegenden Falles waren dic ausgeschicdenen Zuckermengen durchaus keine sehr hohen. Die höchsten $\Lambda$ usscheidungen wurden während des Comas constatirt; sie betrugen $120 \mathrm{~g}$. Während der Behandlung gelang es sogar, die Zuckerausscheidung vorübergehend bis auf $2,5 \mathrm{~g}$ pro die (25. u. 26. 11.) herabzudrücken, d. h. die Kranke practisch zuckerfrei zu machen; gegen Ende der Behandlung machte sich trotz der Haferperiode wiederum ein durch Schwankungen unterbrochener langsamer Anstieg der Zuckercurve geltend. Bemerkenswerth ist, dass die Kohlehydratbilanz während der Hafertage mit einer Kohlehydratzufuhr von $177-140 \mathrm{~g}$ pro die stets positiv war. Dagegen während der Periode mit strenger Diät stets negativ. Auffällig erscheint das sprunghafte Auftreten einer Vermehrung der Zuckerausscheidung bei derselben Diät; so z. B. $9,0 \mathrm{~g}$ gegenüber $33,3 \mathrm{~g}$ (1. 2. und 2. 3. 12.) sowie 16,5 gegen 40,3 und 17,1 gegen $63,5 \mathrm{~g}$ (14.-18. 12.), ohne dass dabei eine wesentlich grössere Urinmenge secernirt wurde.

Auf die relativ grosse Oxalsäureausscheidung während der Hafertage hat der eine von uns (13) berejts an anderer Stelle hingewiesen.

Die Acetonkörperausschoidung lässt bestimmte Rückschlüsse auf die Wirkung der einzelnen Diätformen nicht zu, weil die Differenzen zwischen 
den einzelnen Perioden nur sehr geringe sind, nachdem die Acetonkörper einmal von der abnormen, während des Comas erreichten Höhe auf ein gewisses Minimum herabgegangen sind. Es wurden trotz Natrongaben bis zur Alkalescenz des Urinos stets grössere Mengen derselben ausgeschieden, nur an einzelnen 'Tagen waren geringere Werthe als $10 \mathrm{~g}$ zu verzeichnen. Verhältnissmässig gross erscheint an einzelnen Tagen z. B. am 28. u. 29. 11. und am 3. u. 4. 12. der Antheil des Acetons an den Gesammtacetonkörpern mit 42 pCt. bzw. 35 pCt. trotz einer Gesammtausscheidung in mittlerer Höhe von 14 bis $17 \mathrm{~g}$, cin Verhalten, wie es bei Fall II bereits erörtert ist.

$\mathrm{Zu}$ einigen Bemerkungen veranlassen noch die Gewichtsschwankungen der Kranken. In 15 Tagen nahm dieselbe um $4,7 \mathrm{~kg} \mathrm{zu}$, eine Zunahme, die ca. 13 pCt. des Gewichtes bei der Aufnahme ausmacht. Während dieser ganzen Zeit ist nur einmal eine Urinmenge von 2,1 Liter zu verzeichnen, vielfach erreicht dieselbe nicht einmal die Höhe von 1 Liter. Wenn dies Verhalten schon für eine Wasserretention spricht, so noch mehr die Thatsache, dass Oedeme der Knöchel auftraten und dass mit Schwinden des Hydrops das Körpergewicht binnen 4 Tagen auf 40 Kilo sank, also fast auf das beim Beginne der Beobachtung herunterging. Obwohl bei dem Zustandekommen des Hydrops das toxische Haferödem v. Noorden's eine gewisse Rolle spielen könnte, so handelt es sich hier jedoch im Wesentlichen $\mathrm{um}$ eine Wasserretention durch Natronüberschwemmung des Organismus; denn nach Aussetzen dieser Medication verschwand das Oedem binnen kürzester Frist.

Zum Schluss sei noch einiges Allgemeine und den beschricbenen Fällen Gemeinsame kurz erörtert.

\section{Ammoniakausscheidung.}

Bei mit Hafer behandeltem schwerem Diabetes behält unter Umständen, auch wenn die Acetonkörperausscheidung abnimmt, der Ammoniakgehalt des Urins bäufig die gleiche relative Höhe bei, wie zu der Zeit, wo eine schwere Acidose das Krankheitsbild beherrschte. Einige in dieser Richtung auffallende Daten seien hier kurz erwähnt. Schon in der ersten Mittheilung von v. Noorden (14) sowie in den Beobachtungen von Falta (15) ist vielfach eine solche abnorme Höhe des Ammoniakgehaltes zu constatiren. Am auffallendsten ist diese Erscheinung jedoch bei Lampé (16) (absolutes Absinken des Ammoniaks von $16 \mathrm{~g}$ auf $1,4 \mathrm{~g}$ bei einem relativen Anstieg von 11 auf 40 pCt.). Diese Erscheinung ist indessen nicht constant, vielmehr verläuft in den meisten Fällen die absolute und relative Ammoniakcurve parallel. Unsere Kranken liessen im Allgemeinen eine so abnorme Höhe des Ammoniakgehaltes nicht durchweg erkennen. Es zeigte sich vielmehr, dass die Ammoniakausscheidung sowohl relativ wie absolut abhängig von der Grösse der Acidose sowie der Höhe des Eiweissumsatzes war. Immerhin deuten bei Einschränkung der N-Zersetzung auf $4-6 \mathrm{~g}$ Zahlen von $21 \mathrm{pCt}$. oder $18 \mathrm{pCt}$. Ammoniak-N wie in Fall III trotz gleichzeitiger Natrongaben oder Gleichbleiben der $\mathrm{NH}_{3}$-Ausscheidung trotz Herabgehens der Acidose und N-Zersetzung (für die 2 crsten Perioden von Fall II) auf 
eine Anomalie in der Ammoniakbildung hin, die durch die Acidose allein nicht genügend erklärt scheint. Nur ist bei Bewerthung der procentualen Ammoniakausscheidung darauf Rücksicht zu nehmen, dass dieselbe bei abnorm niedriger N-Zufuhr schon an und für sich normaler Weise vielleicht etwas höher einzuschätzen sein mag. Wenn man nicht annehmen will, dass in solchen Fällen an Stelle der Acetessigsäure und Oxybuttersäure andere Säuren auftreten, so könnte dieses Verhalten darin liegen, dass diese Gruppe schwerer Diabetiker überhaupt nicht im Stande ist, das im Körper entstehende Ammoniak durch Harnstoffbildung unschädlich zu machen. Diese Möglichkeit wird nahe gelegt durch die Erwägung, dass die Leber, in der sich hauptsächlich die Harnstoffsynthese vollzieht, aus den, ketoplastischen Substanzen reichlich Aceton liefert. Somit könnten solche hohen Ammoniakwerthe vielleicht nur als Ausdruck der gestörten Leberfunction aufzufassen sein.

\section{Niederschlagsstickstoff.}

Ferner war die $\mathrm{N}-$ Vertheilung an einzelnen Tagen zu Gunsten des Niederschlags- $\mathrm{N}$ verschoben. So wurden $9,0-10,7 \mathrm{pCt}$. Niederschlags- $\mathrm{N}$ in Fall II beobachtet, im Fall III sogar einmal 12,2 pCt. und 8,6 pCt. J eider konnten aus äusseren Gründen Purinkörperbestimmungen nicht vorgenommen werden, sodass nicht festgestellt wurde, ob nicht letzteren im Wesentlichen die Höhe des Niederschlags-N zuzuschreiben ist. Allerdings stammen die hier angeführten Zablen aus Perioden stickstoffarmer Kost (Gemüse und Hafer), die Purinsubstanzen in wesentlicher Menge nicht enthiclt. Die mittlere Stickstoffausscheidung betrug für diese Tage im ersteren Falle 8,6, im zweiten Falle $6,1 \mathrm{~g} \mathrm{~N}$. Wenn ich einen hohen Purinkörpergehalt von $0,6 \mathrm{~g}$ bis $0,2 \mathrm{~g} \mathrm{~N}$ pro die selbst annehme, so würde dieser nur 2,4 pCt. bezw. 3,2 pCt. des Gesammt-N ausmachen, so dass im ersten Falle von durchschnittlich $9,9 \mathrm{pCt}$. Niederschlags-N 7,5 pCt., im zweiten Falle von durchschnittlich 10,4 pCt. Niederschlags-N 7,2 pCt. auf den Rest-N entfielen. Aehnliche Befunde sind von Mandel und Lusk (17), sowie von v. Noorden (18) selbst beim Diabetes erhoben worden. Allerdings ist, wie auch bei der $\mathrm{N}$-Ausscheidung, bei der Bewerthung dieser Befunde zu erwägen, dass bej der N-Fraction an eine Verschiebung bei vegetabilischer Kost zu denken ist. So hat kürzlich Spiro (19) für Kohlehydratfütterung und Hunger beim Hunde ein ähnliches Verhältniss gefunden. $O b$ indessen nicht doch hier zum Mindesten für den schweren Diabetiker abnorme Vorhältnisse vorliegen, kann erst eine weitere Durchprüfung dieser Frage zeigen. Wenn das besprochene Verhalten der stickstoffhaltigen Bestandtheile auch kein constantes ist [P. F. Richter (20)], so beweist dies an sich noch nichts gegen das Pathologische dieser Erscheinung, denn bei Körpern, die in so geringen Mengen und in so inconstanten Verhältnissen im Urin auftreten, muss man sich auf Schwankungen von vornherein gefasst machen.

\section{Die Glukosurie.}

Was den Verlauf der Zuckercurve anlangt, so ist bemerkenswerth, dass die Kohlehydratbilanz während der Hafertage stets positiv war, auch in Fall II, der sonst bezüglich der Glukosurie keine sehr günstige Reaction 
zeigte. Auch fand eine beträchtliche Zuckerausscheidung in den ersten auf eine Haferkur folgenden Tagen bei den Kranken nicht statt. Auffallend ist dagegen, dass in Fall 111, nachdem eine Woche lang Gemüsediät nach der Haferkur verabreicht war, plötzliche Steigerungen in der Zuckerausscheidung stattfinden, für die eine Ursache in dem diätetischen Vorhalten nicht festzustellen war. So in der Zeit vom 14.-18, 12, wo hintercinander bei nicht wesentlich schwankender Urinmenge und gleichmässiger Diät $16,5 \mathrm{~g}, 30,3 \mathrm{~g}, 17,1 \mathrm{~g}$ und $63, \overline{\mathrm{g}}$ Zucker im Urin beobachtet wurden. Dieses Verhalten liess daran denken, dass vielloicht ein Theil der im Urin nicht erscheinenden Kohlehydrate des Hafers im Körper als Traubenzucker circuliren, deren der diabetische Organismus sich erst nach vielen Tagen entledigt. Es müsste dann allerdings eine erhebliche Steigerung des Blutzuckergehaltes stattinden, da es sieh (vergl. Fall III) um beträchtliche Kohlehydratmengen meist über $100 \mathrm{~g}$ handelt. Die Wasserretention kann dabei eine sehr wesentliche Rolle nicht spiclen, denn bei eincr Gewichtszunahme von 4,7 Kilo und einem hypothetischen Zuckergehalt von 0,2 pCt. würde dies nur $9,4 \mathrm{~g}$ Glukose ausmachen. Wenn man selbst annimmt, dass bei einem Gewicht von $40 \mathrm{~kg}$ und einem Wassergehalt des Körpers von 70 pCt. die ganze im Körper enthaltene Wassermenge an der Zunahme des Zuckergehaltes gleichmässig participire, so würde man bei ciner Retention von $100 \mathrm{~g}$ Kohlehydrat noch immer eine Wrhöhung des Zuekergehaltes der Gewebssäfte um 0,3 pCt. pro T'ag erhalten. Wenn es auch nicht wahrscheinlich ist, dass diese maximale Zuckerretention wirklich stathat, so jst dic Möglichkeit jedenfalls zuzugeben, dass wenn auch nicht die Gesammtmenge, so doch ein Theil der Kohlehydrate in dieser Weise im Körper eine Zeit lang circulirt. Es ist ja auch mehrfach nachgewiesen worden, dass sowohl beim pankreasdiabetischen Hunde [Mohr (21), Mohr und Hesse (22)] wie auch beim Menschen [v. Noorden (23), Naunyn (24), Liefmann und Stern (25)] ein starkes Missverhältniss zwischen Glykämie und Glukosurie bestehen kann. Hicrzu kommt, dass systematische Untersuchungen über den Blutzuckergehalt bei Haferkuren nicht vorliegen. Der anfangs citirte Fall von v. Noorden mit so eklatant günstiger Haferwirkung zeigte trotz zuckerfreien Urins eine mässige Hyperglykämie. Jedenfalls bedarf diese Frage noch weiterer Klärung. Im Uebrigen lassen unsere Beobachturigen den Schluss zu, dass jedenfalls cin Theil des Hafers vom diabetischen Organismus verwerthet wird. Hicrin liegt vielleicht auch dic Erklärung für den Werth des Hafers als antikotoplastischen Factors. Wenn im Fall III sich gegen Ende der Beobachtung ein Ansteigen der Glukosuric bemerkbar macht, so mag dies darin liegen, dass in solchen Fällen mit nur temporärem Lirfolge der Hafer, ähnlich wie die Lävulose, zunäehst als ungewohnter Zellreiz wirkt [Lüthje (26)], bei öfterer Anwondung indessen versagt $^{1}$ ).

1) Da längere Perioden kohlehydratfreier Kost in den vorliegenden Fällen nicht. verabreicht wurden, so konnten wir Rückschlïsse auf die Quellen der 'Juckerbildung im allgemeinen nicht machen. Es sei hier nur kurz auf ein bemerkenswerthes Verhalten des Falles III hingewiesen, bei dem wir den Falta'schen Quotienten (27) D: $5 \mathrm{~N}+\mathrm{K}$ (D Zucker, $\mathrm{N}$ Stickstoffgehalt des Urins, K Kohlehydrat der Nahrung) 


\section{Ketonurie.}

Der Einfluss der Haferkur auf die Acidose tritt in Fall II und III deutlich zu Tage; im ersteren gelingt es, dieselbe ohne Natron auf minimale Werthe herabzudrücken; in Fall III wird allerdings bei reichlicher Alkalizufuhr nicht nur das drohende Coma abgewandt, sondern auch durch fortgesetzte Hafer- und Gemüsediät die Glukosurie vorübergehend auf ein Minimum reducirt. Das Coma, dem diese Kranke noch einmal entging, veranlasst zu einer kurzen theoretischen Bemerkung über sein Wesen sowie über die Wirkungsweise des Hafers. Schon oben ist darauf hingewiesen worden, dass eine Gewichtszunahme von $4,7 \mathrm{~kg}$, die auf Wasserretention durch Alkaligaben in der Hauptsache bezogen wurde, und die $13 \mathrm{pCt}$. des Körpergewichtes ausmacht, mit einer vielleicht nicht ganz unerheblichen Diluirung der Acetonkörper im Organismus gleichzeitig eine Herabsetzung ihrer toxischen Wirkung, die jetzt wohl als feststehend angesehen wird, bewirken muss. Der Kranken ist Natron bis zur Alkalescenz des Urins verabfolgt worden. Dazu wurden $280 \mathrm{~g}$ Natron in der Zeit vom 17.-28. 11. benötigt, die $336 \mathrm{~g}$ 0xybuttersäure entsprechen. Im Urin ausgeschieden wurden während dieser Zeit im ganzen $218 \mathrm{~g}$ dieser Substanz, von denen $72 \mathrm{~g}$ durch das ausgeschiedene Ammoniak und $146 \mathrm{~g}$ durch Alkali neutralisirt sein müssen. Somit würden schätzungsweise $158 \mathrm{~g}$ Natron übrig bleiben, welche dazu gebraucht sein müssen, um die im Körper vorhandene Oxybuttersäure in das Natronsalz überzuführen, wenn man annimmt, dass mit der Neutralisation des Urines das Maximum der thatsächlichen Absättigung der Acetonkörper durch Alkali erreicht wird. Dies würde einem Gehalte der Gewebe von $170 \mathrm{~g}$ Oxybuttersäure entsprechen. Wenn auch diese Zahl sicher zu hoch gegriffen ist, da ein Theil des Natrons sich im Koth findet, ein anderer an die normaler Weise auftretenden Säuren gebunden sein dürfte, und da ferner trotz der Alkaligaben Aceton mit der Athmungsluft entweicht, so ist doch ein Gehalt von $100-200 \mathrm{~g}$ Oxybuttersäure im Körper nichts Seltenes [Magnus-Ijevy) (28]. Selbst wenn man als Minimum der vorhandenen Oxybuttersäure die am Tage des Comas ausgeschiedene Menge von $47 \mathrm{~g}$ annimmt, so entspricht dies noch immer $1,2 \mathrm{~g}$ Oxybuttersäure pro Kilogramm Körpergewicht. Diese Zahlen zeigen, dass jedenfalls trotz des Natrons erhebliche Mengen Oxybuttersäure im Organismus zurückbleiben.

Es besteht hier also trotz Entgiftung der Säuren als solcher und trotz positiver Kohlebydratbilanz in dieser Zeit die Acetonkörperbildung weiterhin fort, mit der die Ausscheidung dieser Körper nicht gleichen

zur Beurtheilung der Zuckerbildung herangezogen haben. Nach einer 9 tägigen Gemüseperiode (9.-18. 12.), wo er 56 betrug, steigt er bei Fleischzulage (18./20. 12.) auf 172, also weit über das theoretische Maximum. Da diese zweite Periode fettärmer wie die ersterwähnte war, so kann zur Erklärung dieses Verhaltens keine Zuckerbildung aus Fett, sondern augenscheinlich nur eine verspätete Ausscheidung längere Zeit retinirter Zuckermengen in Betracht kommen. Dies Verhalten ist eine Illustration dafür, dass es ausserordentlich schwierig ist, beim Diabetiker Rückschlüsse auf die Herkunft des Zuckers aus Relationen, wie dem Falta'schen (quotienten, zu machen. 
Schritt hält. Günstig wirkt vielleicht trotzdem die relativ grosse Wasserretention, auf die ausführlicher Stäubli (29) hingewiesen hat, wodurch die Acetonkörper diluirt und ihre toxische Wirkung gemindert wird. Es fragt sich, ob nicht überhaupt der Wasserverarmung des diabetischen Organismus eine gewisse Rolle bei der Entstehung des Comas zukommt, eine Ansicht, die Rumpf (30) bereits vor Jahren vertreten hat.

Zum Schluss sei kurz noch einmal darauf hingewiesen, dass der Enderfolg bei den beobachteten Kranken insofern paradox erscheint, als die anscheinend mit Erfolg behandelten Fälle II und III in sehr kurzer Zeit nach der Entlassung am Coma zu Grunde gingen; dass dagegen der einer Therapie gar nicht unterzogene zuerst beschriebene Kranke, der in den ungünstigsten sozialen Bedingungen ohne jegliche Diät lebt, bereits über 1 Jahr die Beobachtungszeit überdauert hat.

\section{Zusammenfassung.}

1. Bei den beobachteten Fällen war die Glukosurie während dor Hafertage stets geringer als die Kohlehydratzufuhr.

2. Die Becinflussung der Zuckerausscheidung durch den Hafer war nicht abhängig von der Schwere des Falles.

3. Wahrscheinlich spielt bei der Haferwirkung eine Retention von Kohlehydraten im Blute eine Rolle.

4. Die günstige Einwirkung des Hafers auf die Glukosurie war bei Wiederholung nicht stets die gleiche, selbst da, wo der Zucker zunächst bis auf Spuren aus dem Urin schwand.

5. Trotz prompten Rückganges der Acidose nach Verabreichung des Hafers gelang es nicht, selbst bei hohen Natrondosen, dieselbe völlig zum Verschwinden zu bringen; vielmehr ist anzunehmen, dass trotz vermehrter Ausscheidung Ketonsubstanzen in erheblicher Menge im Körper zurückbleiben.

6. Die Stickstoffvertheilung im Harn liess an einzelnen Tagen eine durch Acidose nicht erklärte Vermehrung des Ammoniakgehaites sowie des dysoxydablen Stickstoffs erkennen. Ob dies Verhalten der letztgenannten N-Fraction für manche Diabetiker charakteristisch oder nur eine Folge $\mathrm{N}$-armer Ernährung ist, ist nicht zu entscheiden.

\section{Literatur.}

1. v. Noorden, Berliner klin. Wochenschr. 1903. Nr. 36 . S. 817.

2. Lampé, Zeitschr. f. phys. u. diät. Therap. Bd. 13. S. 1.

3. Falta, Ergebn. d. inn. Med. u. Kinderheilk. 1908. Bd. 2. S. 74.

4. Lüthje, Therapie d. Gegenw. N. F. 1910. H. 1.

5. Derselbe, Zeitschr. f. klin. Med. 1900. Bd. 39. S. 397 und 1901. Bd. 43. S. 225.

6. Mohr, Zeitschr. f. exp. Pathol. u. Therap. 1906. Bd. 2. S. 467.

7. Falta, ef. Anm. 3.

8. v. Noorden, Med. Klinik, 1909. Nr. 35. S. 1292.

9. Embden u. Schmitz in Abderhalden, Handb. d. biochem. Arbeitsmethoden. Bd. 3. S. 906. 
10. Neubauer, Verhandl, d. Deutschen Congresses f. innere Med. Wiesbaden 1910. S. 566.

11. v. Noorden, Pathol. d. Stoffwechsels. 1907. Bd. 2. S. 89.

12. Spiro, Hofmeister's Beiträge. Bd. 10. S. 272.

13. Jastrowitz, Biochem. Zeitschr. 1910. Bd. 28. S. 34 .

14. v. Noorden.

15. Falta, siehe Anm. 3.

16. Lampé, siehe Anm. 2.

17. Mandel u. Lusk, Deutsches Arch. f. klin. Med. 1904. Bd. 81. S. 472.

18. v. Noorden, Pathol. d. Stoffwechsels. 1907. Bd. 2. S. 91.

19. Spiro, siehe Anm. 12.

20. Richter, P. F., Charitéannalen. 1897. Bd. 22. S. 287.

21. Mohr, Zeitschr. f. exp. Pathol. n. Therap. 1907. Bd. 4. S. 910.

22. Mohr u. Hesse, Ebendas. 1909. Bd. 6. S. 300 .

23. v. No orden, Pathol. d. Stoffwechsels. 1907. Bd. 2. S. $7-8$.

24. Naunyn, Diabetes mellitus. 1906. S. 188/89.

25. Liefmann u. Stern, Biochem. Zeitschr. 1906. Bd. 1. S. 299.

26. Lüthje, siehe Anm. 4 .

27. Falta, Zeitschr. f. klin. Med. 1808. Bd. 65.467 et sq.

28. Magnus-Levy, Ergebn. d. inn. Med. u. Kinderheilk. 1908. Bd.1. S. 352.

29. Stäubli, Deutsches Arch. f. klin. Med. 1908. Bd. 93. S. 107.

30. Rumpf, Zeitschr. f. klin. Med. 1902. Bd. 45. S. 260, sowie Dennstedt und Rumpf, Ebendas, 1906. Bd. 58. S. 84 . 